\title{
Highwire Function at the Drosophila Neuromuscular Junction: Spatial, Structural, and Temporal Requirements
}

\author{
Chunlai Wu, Yogesh P. Wairkar, Catherine A. Collins, and Aaron DiAntonio \\ Department of Molecular Biology and Pharmacology, Washington University School of Medicine, St. Louis, Missouri 63110
}

\begin{abstract}
Highwire is a huge, evolutionarily conserved protein that is required to restrain synaptic growth and promote synaptic transmission at the Drosophila neuromuscular junction. Current models of highwire function suggest that it may act as a ubiquitin ligase to regulate synaptic development. However, it is not known in which cells highwire functions, whether its putative ligase domain is required for function, or whether highwire regulates the synapse during development or alternatively sets cell fate in the embryo. We performed a series of transgenic rescue experiments to test the spatial, structural, and temporal requirements for highwire function. We find that presynaptic activity of highwire is both necessary and sufficient to regulate both synapse morphology and physiology. The Highwire RING domain, which is postulated to function as an E3 ubiquitin ligase, is required for highwire function. In addition, highwire acts throughout larval development to regulate synaptic morphology and function. Finally, we show that the morphological and physiological phenotypes of highwire mutants have different dosage and temporal requirements for highwire, demonstrating that highwire may independently regulate the molecular pathways controlling synaptic growth and function.
\end{abstract}

Key words: highwire; synaptogenesis; synaptic transmission; ubiquitin ligase; neuromuscular junction; Drosophila

\section{Introduction}

Structural and functional synaptic plasticity underlies the refinement of neural circuits during development and the activitydependent modification of neural pathways in the mature brain. highwire (hiw) is positioned to coordinate the morphological and physiological maturation of the synapse, because it restrains synaptic growth and promotes synaptic strength at the Drosophila neuromuscular junction (NMJ) (Wan et al., 2000). Highwire (Hiw) is a huge, neuronal protein whose structure, and likely function, is evolutionarily conserved. The Caenorhabditis elegans homolog rpm-1 regulates synapse morphology (Schaefer et al., 2000; Zhen et al., 2000), the zebrafish homolog esrom is required for normal retinotectal projections (D'Souza et al., 2005), and deficiencies removing the mouse homolog $\mathrm{Phr}$ and neighboring genes have altered NMJ development (Burgess et al., 2004). All hiw homologs are comprised of an $\mathrm{N}$-terminal guanine-nucleotide exchange factor-like domain, two PHR repeats of unknown function, and a C-terminal RING finger that is a putative E3 ubiquitin ligase domain. hiw and its homologs have been proposed to regulate a number of candidate signaling pathways (Scholich et al., 2001; McCabe et al., 2004; Murthy et al., 2004; Nakata et al., 2005).

\footnotetext{
Received June 20, 2005; revised Sept. 8, 2005; accepted Sept. 8, 2005.

This work was supported by National Institute of Neurological Disorders and Stroke Grant NS043171, the Keck Distinguished Young Scholars Program, and the McKnight Scholars Award (A.D.), as well as Paralyzed Veterans of America and the Damon Runyon Foundation (C.A.C.). We thank Sylvia Johnson, Xiaolu Sun, and Scott Portman for excellent technical assistance and Richard Daniels for helpful comments.

Correspondence should be addressed to Dr. Aaron DiAntonio, Department of Molecular Biology and Pharmacology, Campus Box 8103, 660 South Euclid, Washington University School of Medicine, St. Louis, M0 63110. E-mail: diantonio@wustl.edu.

DOI:10.1523/JNEUROSCI.2532-05.2005

Copyright $\odot 2005$ Society for Neuroscience $\quad 0270-6474 / 05 / 259557-10 \$ 15.00 / 0$
}

The requirements for hiw function are unknown. Spatially, the hiw transcript is abundant in neurons, but its functional requirement could be either presynaptic or postsynaptic. Understanding how hiw controls synaptic development requires knowing where it functions. Structurally, Hiw is likely an ubiquitin ligase. It contains a RING motif characteristic of ubiquitin ligases (Joazeiro and Weissman, 2000), interacts with the deubiquitinating protease fat facets (DiAntonio et al., 2001), and RPM-1 binds to an E3 ubiquitin ligase complex (Liao et al., 2004). Although Hiw may be a ligase, there is no evidence that this is necessary for its function. Mutants have been identified in flies and worms that are predicted to disrupt the RING domain, but it is not known whether these mutant proteins are expressed (Schaefer et al., 2000; Wan et al., 2000; Zhen et al., 2000). Therefore, the ligase activity may be required for none, some, or all hiw function. Temporally, the hiw phenotype is apparent in the embryo. Hence, hiw may act early as a cell fate determinant setting the synaptic growth rate, or it could function throughout development to modify an ongoing synaptogenic signal. To investigate the tissue, structural, and temporal requirements for hiw function, we have undertaken a structure/function analysis.

We generated new hiw alleles that produce no full-length Hiw protein, antisera that recognize Hiw, and both wild-type (WT) and epitope-tagged hiw transgenes. We find that, although Hiw can localize to synapses, it is not a periactive protein as reported previously (Sone et al., 2000; Wan et al., 2000). We demonstrate that presynaptic hiw function is both necessary and sufficient for normal synapse morphology and physiology. In addition, the RING domain is required for hiw function, and transgenic hiw with a disrupted RING motif is a potent dominant negative. Finally, hiw acts throughout larval development to regulate synaptic structure and transmission. These studies support a model of 
Hiw as a presynaptic ubiquitin ligase acting during synaptic maturation to regulate pathways controlling both synaptic morphology and transmission.

\section{Materials and Methods}

\section{Genetics}

Cloning of hiw full-length cDNA. To generate the $15.7 \mathrm{~kb}$ full-length hiw cDNA, we cloned four overlapping cDNA fragments spanning the entire hiw ORF and then sequentially ligated individual fragments together. In brief, total RNA was extracted from late Drosophila embryos and reverse transcription (RT) reactions were performed using four gene-specific reverse primers, located immediately downstream of $K p n I$ (3044), XhoI (7253), EcoRI (10720), and the stop codon, respectively. Using the RT reactions as templates, we performed PCR to obtain four sequential hiw cDNA fragments: 5' end to KpnI (3044), KpnI (3044) to XhoI (7253), Xho I (7253) to EcoRI (10720), and EcoRI (10720) to 3' end. An NotI site and an $X b a I$ site were introduced into the $5^{\prime}$ end and the $3^{\prime}$ end, respectively, to facilitate subcloning. These four cDNA fragments were then sequentially subcloned into modified pGEM-T easy vector (Promega, Madison, WI) to generate the $15.7 \mathrm{~kb}$ full-length hiw cDNA. The final cDNA fragment was sequenced to completion.

Site-directed mutagenesis. To disrupt the function of Hiw RING finger domain, C4991 and C4994, the first two Cys in the RING structure were changed to Ser, using the GeneEditor in vitro Site-Directed Mutagenesis System (Promega).

Transgenic constructs. The wild-type hiw cDNA was subcloned into the pUAST vector (Brand and Perrimon, 1993) and the pUAS-EGFP (enhanced green fluorescent protein) vector (Parker et al., 2001), respectively, to generate a UAS-Hiw transgene without tag and an N-terminalfusion UAS-GFP-Hiw transgene. The same wild-type cDNA and the cDNA carrying point mutations in the RING finger domain of Hiw were also subcloned into a pUAS-TAP vector (derivates from a TAP construct provided by EUROSCARF, Frankfurt, Germany), respectively, to generate another two transgenes: N-TAP-Hiw (NT-Hiw) and N-TAPHiw $\Delta$ RING (NT-Hiw $\Delta$ RING) (Rigaut et al., 1999). Transformants were generated by standard techniques.

Fly stocks. Flies were maintained in $25^{\circ} \mathrm{C}$ on normal food, unless noted otherwise. The following strains were used in this study: Canton S (wild type), elav Gal4 (neuron specific) (Yao and White, 1994), BG380 Gal4 (neuron specific) (Budnik et al., 1996), ELAV-GeneSwitch (Osterwalder et al., 2001), G7 Gal4 and 24B Gal4 (muscle specific), and six hiw ND alleles, ND8, ND9, ND17, ND36, ND42, and ND69, generated in an ethylmethane sulfonate screen for recessive enhancers of the synaptic morphology phenotype caused by faf overexpression (Wan et al., 2000; DiAntonio et al., 2001).

Excision mutations in hiw. Excision mutations in hiw were made using a P-element-hopping strategy, as described previously (Petersen et al., 1997). Two deletions, hiw ${ }^{\Delta N}$ and $h i w^{\Delta C}$, that respectively remove the $\mathrm{N}$-terminal and C-terminal genomic regions of hiw, were identified.

RU486-GeneSwitch rescue experiments. The RU486-GeneSwitch system was administrated according to Osterwalder et al. (2001) and Roman et al. (2001). Flies used in the GeneSwitch experiments were kept at $18^{\circ} \mathrm{C}$ to reduce the very low transcriptional activity of the GeneSwitch protein in the absence of RU486. Female virgin flies (hiw ${ }^{N D 8}$; ELAV-GeneSwitch) were fed on 2\% sucrose with $500 \mu \mathrm{M}$ RU486 (Mifepristone; Sigma, St. Louis, MO) or $2 \%$ sucrose without RU 486 for $2 \mathrm{~d}$ before mating. Females prefed with RU486 were crossed to the males carrying the UAS-GFP-Hiw transgene on fly food containing $20 \mu \mathrm{g} / \mathrm{ml} \mathrm{RU} 486$, to induce the constitutive expression of GFP-Hiw from embryos to third-instar larvae. Females prefed without RU486 were also crossed to the UAS-GFP-Hiw males on the fly food containing no RU486 as control. To induce the expression of GFP-Hiw after embryogenesis, larvae just hatched from the non-RU486 crosses were manually transferred from non-RU486 food to RU486 food. Third-instar male larvae from all of the crosses were analyzed for rescue of the hiw morphological and physiological phenotypes.

\section{Immunochemistry}

Antibodies. The rabbit anti-Hiw antibody HIW2b was raised against a 14-residue peptide at the N-terminal region of the predicted highwire gene product (-RRGEDGDSSKNRRQ) by Zymed (San Francisco, CA), affinity purified, and used at 1:250 dilution for immunoblot. HIW2b does not work for immunohistochemistry. The rabbit anti-DVGLUT was described previously (Daniels et al., 2004) and used at 1:5000 dilution for both immunoblot and immunohistochemistry. The rabbit antiGFP antibody was a gift from Pam Silver (Harvard Medical School, Boston, MA) (Seedorf et al., 1999). The mouse monoclonal NC82 (gift from Erich Buchner, Theodor Boveri Institut, Würzberg, Germany) stains active zones (Marrus et al., 2004) and was used at a 1:100 dilution. The mouse anti-cysteine-string protein (CSP) antibody was kindly provided by Dr. Zinsmaier and used at 1:100 dilution (Zinsmaier et al., 1990). The monoclonal antibody 6H4 (Wan et al., 2000) and the anti-fasciclin II (FasII) (1D4) antibody developed by Corey Goodman (Renovis, San Francisco, CA), as well as the $\beta$-tubulin monoclonal antibody (E7) developed by Michael Klymkowsky (University of Colorado, Boulder, CO) were obtained from Developmental Studies Hybridoma Bank, developed under the auspices of the National Institute of Child Health and Human Development and maintained by the Department of Biological Sciences of The University of Iowa (Iowa City, IA) and used at 1:25, 1:5, and $1: 1000$, respectively. The mouse monoclonal $6 \mathrm{H} 4$ was tried with a variety of fixation conditions, and all gave similar results.

Immunoblot. Approximately 20 larval brains of each genotype were collected in a $1.5 \mathrm{ml}$ microcentrifuge tube and kept at $-80^{\circ} \mathrm{C}$ until homogenization. Each brain sample was homogenized in an ice bath with $0.1 \mathrm{ml}$ of homogenization buffer (67 mm Tris- $\mathrm{HCl}, \mathrm{pH} 8.0,67 \mathrm{~mm} \mathrm{NaCl}$, 2 м urea, 1.3\% SDS, 2 mм DTT, and 1 mм EDTA) using strokes of a pellet pestle (Kimble/Kontes, Vineland, NJ). Immediately after adding SDS loading buffer, the homogenate was boiled for $5 \mathrm{~min}$ and then loaded onto 5\% SDS-PAGE gel. Samples for detecting the endogenous Hiw were probed on Western blots using primary antibodies to Hiw (HIW2b at $1: 250)$ and DVGLUT (1:5000) or $\beta$-tubulin (1:1000). Samples for detecting the transgenic NTAP-Hiw proteins were probed on immunoblot with a peroxidase-antiperoxidase complex (Sigma P-2026) at 1:4000 dilution. Quantification of the total Hiw levels on Western blots was performed using the GS-710 Calibrated Imaging Densitometer (Bio-Rad, Hercules, CA), and the intensity of the Hiw bands was normalized against the control $\beta$-tubulin bands.

Immunohistochemistry. Wandering third-instar larvae were dissected in ice-cold PBS and fixed in either Bouin's fixative (1:5:15 ratio of acetic acid/formalin/picric acid) or $5 \%$ formaldehyde. Larvae were then washed in PBS containing $0.1 \%$ Triton-X (PBX) and blocked for $30 \mathrm{~min}$ at room temperature in $5 \%$ normal goat serum in PBX. Primary antibody was diluted in $\mathrm{PBX}$ and incubated overnight at $4^{\circ} \mathrm{C}$ or at room temperature for $1 \mathrm{~h}$. Cy3- and Alexa-488-conjugated secondary antibodies (Jackson ImmunoResearch, West Grove, PA) were used at 1:1000 for $1 \mathrm{~h}$ at room temperature. After staining, larvae were equilibrated in $70 \%$ glycerol in PBS and mounted with VectaShield (Vector Laboratories, Burlingame, CA).

Imaging and analysis. Larvae were imaged on a Nikon (Tokyo, Japan) $\mathrm{C} 1$ confocal microscope. All images were acquired in third-instar wildtype, mutant, and rescued larvae that had been simultaneously stained. The gain was chosen as the maximum gain that did not saturate the signal for each sample. A complete $\mathrm{z}$-stack was acquired for each NMJ and rendered as a maximum projection, except for the analysis of the colocalization of GFP-Hiw and other synaptic markers, in which single-layer images were acquired. All quantifications were done at muscle 4 in segments A2-A4 while blinded to genotype. The muscle sizes for all genotypes were similar. The number of type I boutons and the number of branch points of type I boutons were counted after anti-DVGLUT and anti-FasII staining. A synaptic branch was defined as an arborization containing at least two type I boutons. Synaptic span was calculated by dividing the length of type I synapses by the length of muscle. The length of synapses and muscle were measured by an ocular micrometer (Nikon). Quantification of bouton size was done using MetaMorph software (Universal Imaging, West Chester, PA). The sizes of $20 \sim 80$ individual type I boutons in each muscle 4 were measured, and an average of 16 cells were measured for each genotype. Statistical analysis was performed and graphs were generated in Origin 7.0 (Origin Lab, Northampton, MA). Each sample is compared with other samples in the group using ANOVA, 
with $n$ for each condition described in Figures 1-7. All histograms are shown as mean \pm SEM.

\section{Electrophysiology}

Intracelluar electrophysiological recordings were performed as described previously (Marrus and DiAntonio, 2004; Marrus et al., 2004). Both spontaneous miniature excitatory junction potentials (mEJPs) and evoked EJPs were recorded in $0.45 \mathrm{~mm} \mathrm{Ca}^{2+}$ Stewart saline (HL-3) (Stewart et al., 1994). At least 60 consecutive events were measured per cell using MiniAnal (Synaptosoft, Decatur, GA) and averaged to determine the mean $\mathrm{mEJP}$. Events with a slow rise time course were rejected as artifacts from neighboring electrically coupled muscle cells. Evoked EJPs were recorded by stimulating the cut end of the segmental nerve with a brief ( $1 \mathrm{~ms}$ ) depolarizing pulse. Quantal content was estimated by dividing the mean EJP by the mean mEJP. For all genotypes, the mean resting potentials and input resistances were very similar, with resting potentials of -74 to $-66 \mathrm{mV}$ and input resistances of 5.7-6.6 M $\Omega$.

\section{Results}

Generation and characterization of highwire null mutations

A large number of highwire alleles have been described that are viable and that result in morphological overgrowth of synapses and deficits in neurotransmitter release. Many of these mutants are nonsense alleles that may generate truncated protein (Fig. 1a). Because Highwire is a huge protein (5233 amino acids) with many domains, we wished to determine whether these mutants expressed truncated proteins as a prelude to a structure/function analysis of hiw. To detect Hiw protein, we raised a polyclonal antibody (HIW2b) against a peptide in the Hiw N-terminal region. On immunoblots of brain extract from wild-type thirdinstar larvae, HIW2b sera recognizes a single band of $\sim 570 \mathrm{kDa}$, as expected for wild-type Hiw. In brain extracts from the nonsense alleles ND8, ND9, ND42, and ND69 (Wan et al., 2000), HIW2b recognizes a truncated protein of the predicted size (Fig. $1 b)$. Hence, these alleles do express truncated protein. In addition, we characterized two mutants, ND36 and ND 17, for which the molecular lesion has not been identified, and find that they also express truncated protein and propose that they both have premature stop codons at approximately amino acid 2700. In addition to the truncated protein, each nonsense allele also expresses some full-length Hiw protein (Fig. 1b). This is most likely attributable to readthrough of the nonsense mutation. Hence, the previously identified hiw alleles are not protein null and may not be functional nulls.

To determine the null phenotype of hiw, we generated excision mutants by imprecise excision of P-elements in the hiw gene. The hiw locus spans $>50 \mathrm{~kb}$ and contains two internal genes (CG5541 and CG32594) in a large intron (Fig. 1a). For the mutagenesis, we used two P-element insertions, P[BG0215] and P[EP1305], that had been identified by the Drosophila genome project. BG0215 is located between the first and the second exons at the $5^{\prime}$ end of the gene. By the imprecise excision of BG0215, we generated a large excision, $h i w^{\Delta N}$, which deletes the genomic region encoding the first 2784 amino acids of hiw, as well as both of the genes contained within the large excision (Fig. 1a). Extracts from hiw ${ }^{\Delta N}$ brains show no Hiw protein on immunoblots, confirming the presence of the mutation and demonstrating the specificity of the HIW2b sera. We cannot exclude the possibility that hiw ${ }^{\Delta \mathrm{N}}$ could express a truncated C-terminal protein. In addition to this large $\mathrm{N}$-terminal deletion, we generated a C-terminal deletion by the imprecise excision of EP1305, which is located at the $3^{\prime}$ end of the large intron. This excision, hiw ${ }^{\Delta C}$, removes the DNA encoding $>2000$ amino acids in the $3^{\prime}$ half of the gene. Immunoblot analysis shows a band of $\sim 300 \mathrm{kDa}$, which is the predicted size for a protein that only includes the a

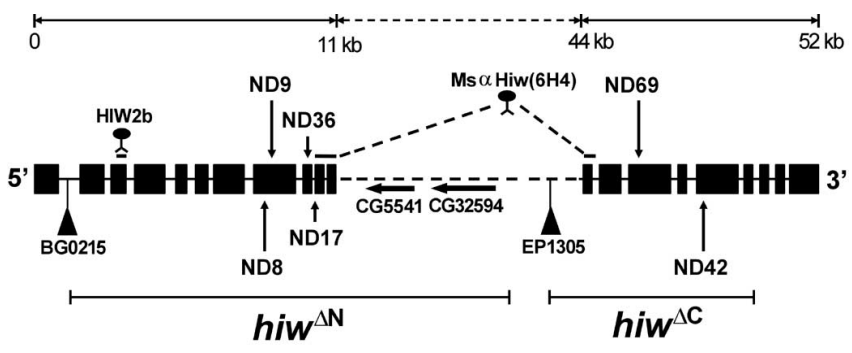

b

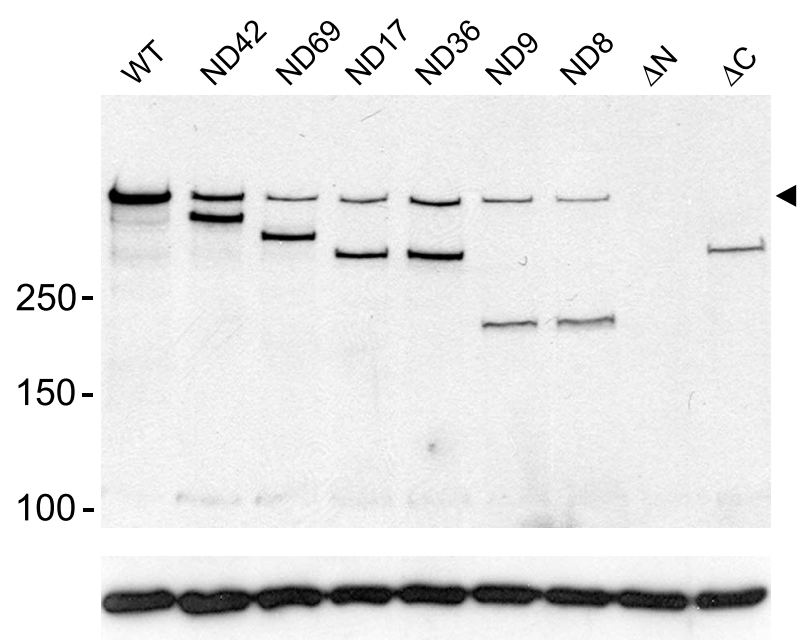

Figure 1. Western blot reveals Hiw expression profile in wild-type and hiw mutant alleles. $\boldsymbol{a}$, The gene structure of hiw. Black boxes indicate exons. Alleles ND8, ND9, ND42, and ND69 have nonsense mutation at amino acid 1930,2001,3173, and 3812, respectively. Molecular lesions of alleles ND17 and ND36 have not been identified by sequencing; however, they are proposed to be nonsense mutations at amino acid proximately 2700 (indicated by arrows). The locations of two P-element alleles, BG0215 and EP1305, are indicated. These two P-elements were used to generate two deletion mutants, hiw $^{\Delta N}$ and $h i w^{\Delta C}$ (the molecular lesions of which are indicated), by imprecise excision. The locations of the antigens that are used to generate the rabbit polyclonal antibody HIW2b and the monoclonal antibody $6 \mathrm{H} 4$ are indicated. Two genes, $\mathrm{CG} 5541$ and (G32594, are located in the large intron (indicated by a dotted line), which spans $\sim 33 \mathrm{~kb}$. $\boldsymbol{b}$, Expression of the endogenous Hiw protein in wild-type and individual hiw alleles was analyzed by immunoblot with antisera HIW2b, as shown in the top panel. Numbers to the left are molecular masses (in kilodaltons). The arrowhead points to the full-length Hiw band at $\sim 570$ $\mathrm{kDa}$. The same blot was probed for DVGLUT as a loading control, as shown in the bottom panel.

$\mathrm{N}$-terminal half of the protein (Fig. 1b). Hence, this excision likely generates a mutant protein including none of the amino acids encoded downstream of the large intron. These two new alleles are likely nulls; they delete large regions of the protein and are the first hiw alleles to make no full-length protein. Despite the dramatic disruption to the hiw gene in the hiw ${ }^{\Delta N}$ and hiw ${ }^{\Delta C}$ alleles, both are homozygous viable and show very similar phenotypes to the previously described hiw alleles (see below).

\section{hiw is required presynaptically to regulate synaptic growth}

Transgenic rescue of mutant phenotypes is a powerful technique for determining the spatial, temporal, and structural requirements for gene function. To apply this analysis to highwire, we generated a full-length, hiw cDNA encoding the entire 5233 amino acid protein (see Materials and Methods). We then created transgenic flies in which the hiw cDNA is under the control 
of the yeast UAS promoter (UAS-Hiw). To facilitate studies of hiw localization, we fused GFP to the $\mathrm{N}$ terminus of full-length hiw to generate UAS-GFP-Hiw transgenic flies.

Before initiating rescue experiments, we compared the phenotypes of preexisting hiw mutants to the newly generated excision mutants. Morphological analysis at the NMJ of hiw ${ }^{\Delta N}$ and hiw $^{\Delta C}$ reveals that both mutants show a dramatic synaptic overgrowth phenotype that is indistinguishable from that of ND8 (a strong hiw nonsense allele) (Fig. 2a). The hiw phenotype is apparent at every type I synapse, which is the primary glutamatergic, excitatory synapse at the NMJ. To quantify this phenotype, we restricted our analysis to the NMJ of a single, identified motoneuron, MN4-1b (Hoang and Chiba, 2001), which forms the type Ib innervation onto muscle 4 . We quantified four metrics of synaptic structure: bouton number, number of branch points, synaptic span (the extent of the muscle covered by the synapse), and bouton size. The three mutants have very similar effects on all of these parameters. We observed a fivefold increase in the numbers of both synaptic bouton and branch points in hiw ${ }^{\Delta N}$, hiw ${ }^{\Delta C}$, and $h i w^{N D 8}$ when compared with the wild-type NMJ (Fig. 2b,c). The synaptic span in all three mutants is also increased from $15 \%$ in wild type to $\sim 40 \%$ (Fig. $2 d$ ). Although there is an increase in the number of synaptic boutons, all three mutants show a $70 \%$ decrease in the average bouton size compared with wild type (Fig. $2 e$ ). Although all quantification was performed on a single, identified motoneuron, all type I NMJs show qualitatively similar phenotypes. Hence, the excision mutants of hiw have essentially identical synaptic phenotypes to the previously identified nonsense allele $h_{i w}{ }^{N D 8}$, and hemizygous excision mutants look similar to transheterozygotes of hiw ${ }^{N D 8}$ and excision mutants. Because the phenotypes are so similar, and because $h i w^{N D 8}$ is much less likely to disrupt the two genes in the hiw intron, CG5541 and CG32594, than the excision mutants are, we performed our transgenic rescue experiments in an hiw ${ }^{N D 8}$ background.

We first wished to determine whether hiw is required presynaptically, postsynaptically, or both, to restrain synaptic growth. To express Hiw in the presynaptic motoneuron, we drove the UAS-hiw transgene with elav-Gal4, a pan-neural Gal4 driver, to generate pre-Hiw ( hiw $^{N D 8} / \mathrm{Y}$; UAS-Hiw/+; elav Gal4/+) rescue larvae. To assess rescue, we again quantified bouton number, synaptic branching, synaptic span, and bouton size at the MN4-Ib NMJ onto muscle 4. All of these metrics of synaptic overgrowth are rescued in the pre-Hiw larvae (Fig. 2). For bouton number, synaptic branching, and synaptic span, the rescue is essentially complete ( $p>0.5$; pre-Hiw vs WT) (Fig. $2 b-d$ ); for bouton size, there is robust rescue, but still a slight deficit compared with wild type (Fig. 2e). Presynaptic expression of a GFPtagged Hiw also rescues the synaptic overgrowth phenotypes of $h_{i w}{ }^{N D 8}$ (data not shown). Although we only quantified NMJs onto muscle 4, all type I NMJs show qualitatively similar rescue. In addition, all hiw alleles tested, including $h i w^{\Delta N}$ and $h i w^{\Delta C}$, are rescued by presynaptic expression of hiw (data not shown). When Hiw is expressed postsynaptically from the muscle-specific driver G7-GAL4, no rescue of the hiw morphological phenotype is observed for the following: bouton number [WT, $25.3 \pm 1.7$ $(n=24)$; hiw ${ }^{N D 8}, 106.4 \pm 2.9(n=23)$; post-Hiw $\left(h_{i w}{ }^{N D 8} / Y\right.$; UAS-Hiw/G7 Gal4), $109.9 \pm 7.3(n=24)]$, number of branch points [WT $2.5 \pm 0.2(n=24) ; h_{i w}^{N D 8}, 9.3 \pm 0.5(n=23)$; post-Hiw, $9.5 \pm 0.6(n=24)$ ], or synaptic span [WT, $16.7 \pm$ $0.9 \%(n=24)$; hiw $^{\text {ND8 }}, 42.3 \pm 2.3 \%(n=23)$; post-Hiw, $39.7 \pm$ $2.4 \%(n=24)]$. These data demonstrate that hiw is required in the presynaptic motoneuron, and is not required in the postsynaptic muscle, to regulate synaptic growth at the Drosophila NMJ. a

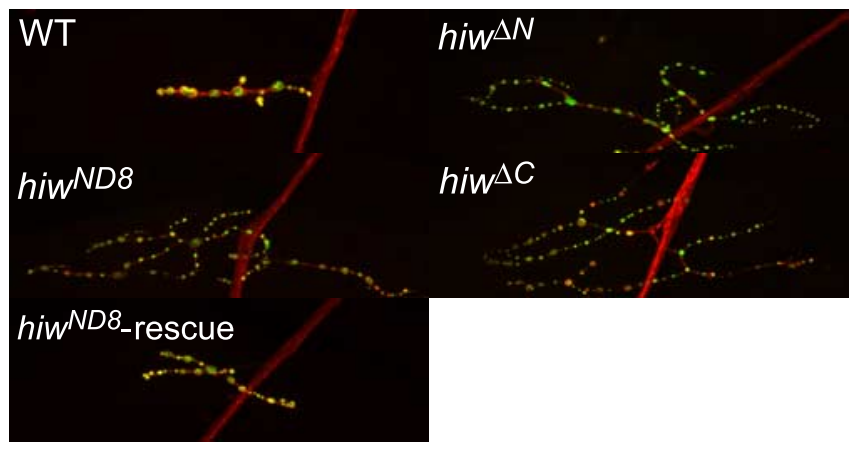

b

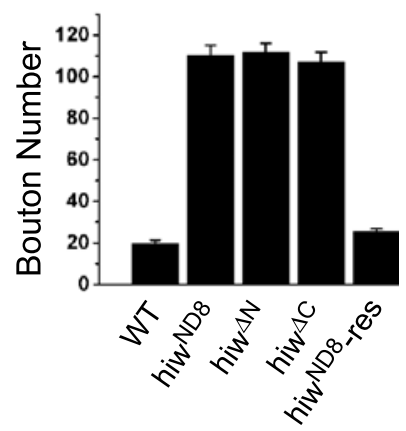

C

d

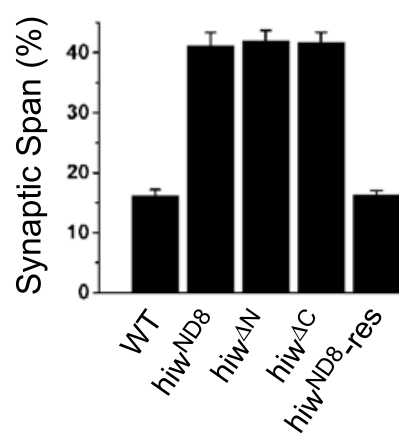

e

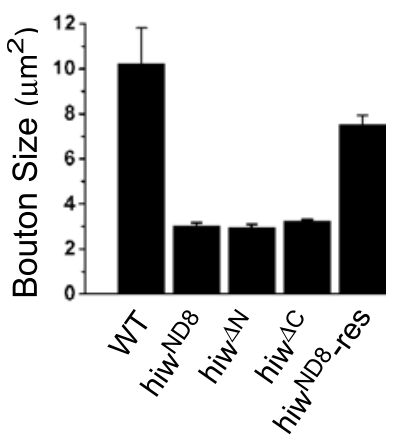

Figure 2. Presynaptic expression of Hiw rescues morphological defects in hiw mutants. $\boldsymbol{a}$ Representative confocal images of muscle 4 synapses costained with DVGLUT (green) and Fasll (red) in WT (elav Gal4/+), hiw ${ }^{N D 8}$, hiw ${ }^{\Delta N}$, hiw ${ }^{\Delta C}$, and hiw ${ }^{N D 8}$-rescue (hiw ${ }^{N D 8} / Y$; UAS-Hiw/+; elav $\mathrm{Gal} / \mathrm{/}+$ ) third-instar larvae. All hiw alleles were out-crossed with wild-type flies. $\boldsymbol{b}-\boldsymbol{e}$, Quantification of bouton number $(\boldsymbol{b})$, number of branch points $(\boldsymbol{c})$, synaptic span $(\boldsymbol{d})$, and bouton size (e) in WT, hiw ${ }^{N D 8}$, hiw ${ }^{\Delta N}$, hiw $^{\Delta C}$, and hiw ${ }^{N D 8}$-rescue third-instar larvae. For bouton number, the number of branch points and synaptic span are $n=30,30,26,37$, and 51 cells, respectively; for bouton size, $n=15,15,21,20$, and 18 cells, respectively. The excision mutants of hiw, hiw ${ }^{\Delta N}$, and hiw $^{\Delta c}$ have essentially identical synaptic phenotypes to the nonsense allele $\operatorname{hiw}^{N D 8}$ ( $p>0.5$ among hiw ${ }^{N D 8}$, hiw ${ }^{\Delta N}$, and $h i w^{\Delta C}$ ). All three hiw alleles are significantly different from WT in all parameters measured ( $p \ll 0.001)$. The hiw morphological defects are rescued by presynaptic expression of Hiw in all parameters measured ( $p \ll 0.001)$. Error bars represent SEM.

\section{Localization of Highwire}

While attempting to characterize the localization of Hiw protein in various hiw alleles, we noticed that staining with the previously described monoclonal antibody (6H4) to Hiw (Wan et al., 2000) does not disappear in any hiw mutants, including the excision alleles. The monoclonal was raised to a 136 amino acid fusion protein that spans the large intron. The hiw ${ }^{\Delta N}$ allele deletes the $\mathrm{N}$-terminal 100 amino acids of this fusion protein, whereas the 

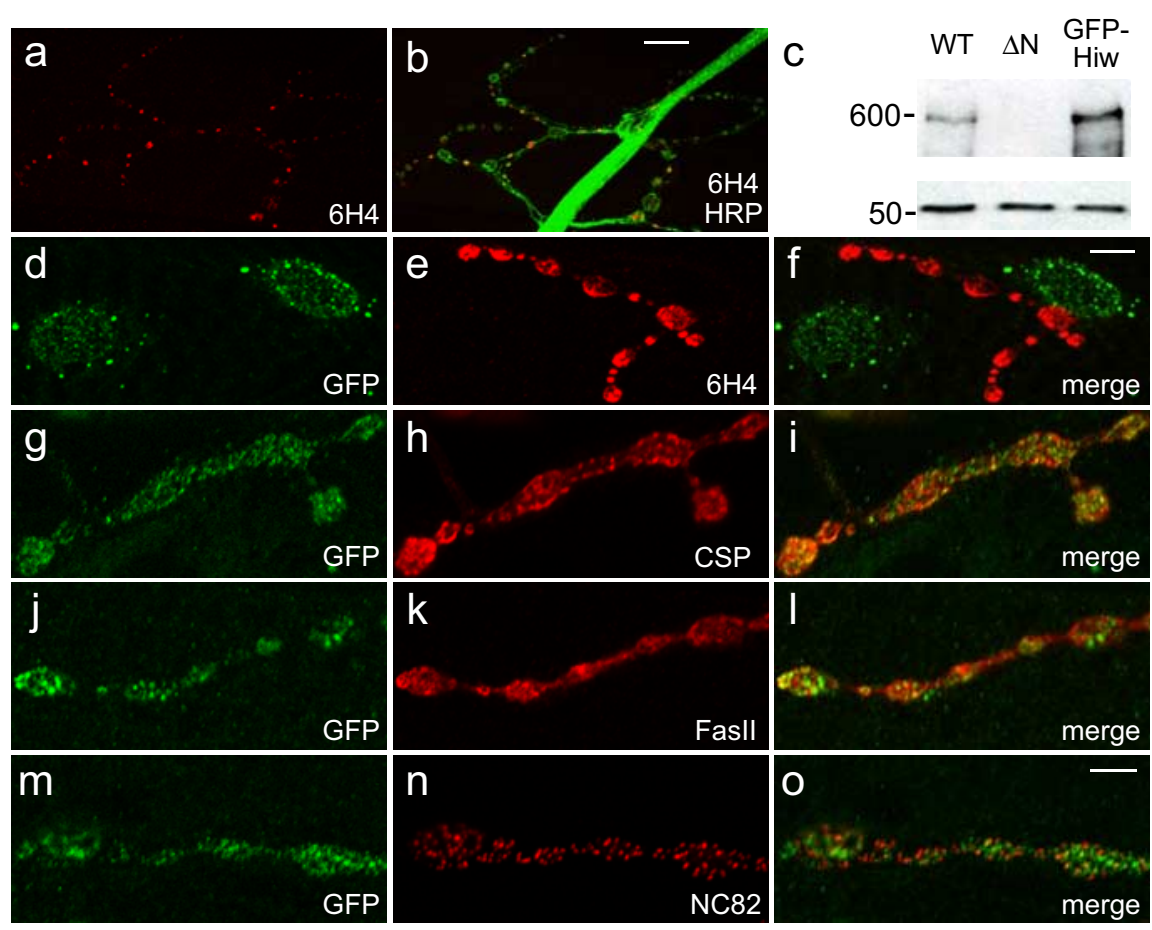

Figure 3. Expression of Hiw. $\boldsymbol{a}, \boldsymbol{b}$, synaptic staining with the mouse Hiw antibody (6H4) remains in the hiw ${ }^{\Delta N}$ deletion mutant, as shown in the representative confocal images of muscle 4 synapses stained with $6 \mathrm{H} 4$ alone (a) or costained with $6 \mathrm{H} 4$ (red) and anti-HRP (green) $(\boldsymbol{b})$. $\boldsymbol{c}$, Expression of the total Hiw protein in the brains of WT, hiw ${ }^{\Delta N}(\Delta N)$, and GFP-Hiw overexpression (GFP-Hiw) larvae was analyzed by immunoblot with antisera HIW2b, as shown in the top panel. Neuronal expression of GFP-Hiw was driven by ELAV-GeneSwitch with RU486 in a wild-type background. The blot in the bottom panel was probed for $\beta$-tubulin as a loading control. Numbers to the left are molecular masses (in kilodaltons). $\boldsymbol{d}$-f, Misexpressed GFP-Hiw in muscle is not recognized by $6 \mathrm{H} 4$, as shown in the representative confocal images of muscle 4 synapses double stained with anti-GFP and $6 \mathrm{H} 4$. The muscle expression of GFP-Hiw was driven by 24B Gal4 driver. $\boldsymbol{g}$ - $\mathbf{0}$, The localization of neuronally expressed GFP-Hiw at the synapse was analyzed by double staining with anti-GFP and synaptic vesicle marker CSP $(\boldsymbol{g}-\boldsymbol{i})$, anti-GFP and periactive zone marker Fasll (j-I), or anti-GFP and active zone marker NC82 ( $\boldsymbol{m}-\boldsymbol{0}$ ). Scale bars: (in $\boldsymbol{b}) \boldsymbol{a}, \boldsymbol{b}, 25 \mu \mathrm{m}$; (in $\boldsymbol{f}) \boldsymbol{d}-\boldsymbol{f}, 13 \mu \mathrm{m}$; (in $\mathbf{0}) \boldsymbol{g}-\mathbf{0}$, $5 \mu \mathrm{m}$.

$h i w^{\Delta C}$ allele deletes the C-terminal 36 amino acids. As such, staining with this monoclonal should have disappeared in one or both of these alleles. Instead, clear synaptic staining with the monoclonal persists at the NMJ and in the neuropil in both excision mutants and in all other hiw alleles (Fig. $3 a, b$ and data not shown). In addition, the monoclonal is not able to recognize Hiw protein that is misexpressed in muscle (Fig. $3 d, e$ ). As such, it is very unlikely that the periactive zone staining pattern seen with this monoclonal reflects the presence of Hiw protein (Sone et al., 2000; Wan et al., 2000).

In an effort to determine the actual localization of Hiw, we generated 10 different sera against peptides and fusion proteins from Hiw. Although the HIW2b sera described above is effective on Western blots, none of the sera work for immunocytochemistry. As an alternative, we analyzed the localization of the fulllength, GFP-tagged UAS-GFP-Hiw transgene that rescues the hiw mutant phenotype. When expressed by a pan-neuronal Gal4 driver, the levels of GFP-Hiw are $\sim 2.8$-fold higher than endogenous Hiw (Fig. 3c). This overexpressed transgene localizes to synapses as shown by costaining with the synaptic vesicle marker CSP (Fig. $3 g-i$ ), periactive zone marker FasII (Fig. $3 j-l$ ), and the active zone marker NC82 (Fig. $3 m-o$ ). Although GFP-Hiw is clearly present at the synapse, it does not extensively colocalize with any of these three markers, although there are occasional regions of overlap. The GFP-Hiw is present diffusely throughout synaptic boutons and often forms puncta within the bouton.
There is no indication of the honeycomb pattern characteristic of the periactive zone localization previously described for Hiw using the monoclonal antibody (Sone et al., 2000; Wan et al., 2000). We also detect GFP-Hiw in axons and cell bodies, although the staining for GFP-Hiw is much stronger at the NMJ than in the preterminal axon. We see no evidence for nuclear or endoplasmic reticulum expression in neurons, as has been reported for protein associated with Myc (PAM) in mammalian cell lines (Guo et al., 1998; Pierre et al., 2004). Results with this overexpressed epitope-tagged Hiw are consistent with the presence of endogenous Hiw at the synapse.

\section{The putative E3 ligase RING domain is essential for Hiw function}

Having addressed spatial requirements for Hiw function, we next focused on structural requirements for hiw activity. The current model for Hiw function posits that it acts as an ubiquitin ligase via its very C-terminal RING finger domain. Although Hiw may be an E3 ligase, it is unknown whether this activity is necessary for some or all of the function of Hiw. To test the requirement for the RING domain for the function of Hiw in vivo, we mutagenized the first two cysteine residues (C4991 and C4994) in the RING finger domain to serine and generated transgenic flies (UAS-Hiw $\Delta$ RING). Previous studies have demonstrated that this alteration to the RING motif abolishes activity in this class of E3 ligases (Lorick et al., 1999). First, we tested the expression of Hiw $\Delta$ RING by immunoblot and found that the mutant transgenic protein is present at very similar levels to wild-type transgenic Hiw when expressed from a neuronal Gal4 driver (Fig. $4 b)$. This Hiw $\Delta$ RING protein fails to rescue any of the synaptic morphology phenotypes of the hiw ${ }^{N D 8}$ mutant (Fig. 4a,c-f). The failure of Hiw $\Delta$ Ring to rescue is not likely caused by a gross alteration in protein folding and stability because the protein can act as a dominant negative when overexpressed in a wild-type background (described below). These data demonstrate that a functional RING finger domain is essential for Hiw-dependent regulation of synaptic growth and support the model that a key function for Hiw is to act as an ubiquitin ligase.

\section{Rescue of the hiw electrophysiological phenotypes}

In addition to synaptic morphology phenotypes, all hiw alleles show reduced strength of synaptic transmission at the NMJ. Decreases in EJPs in the mutant are attributable to (1) a modest decrease in quantal size (response to a single vesicle) and (2) a substantial reduction in quantal content (number of vesicles released by the nerve) (Wan et al., 2000; DiAntonio et al., 2001). To assess whether the spatial and structural requirements for hiw are the same for both its morphological and physiological phenotypes, we expressed wild-type Hiw and Hiw $\Delta$ RING in motoneurons of hiw ${ }^{N D 8}$ mutants and assayed synaptic transmission. We performed intracellular recordings and analyzed both spontane- 
ous and evoked neurotransmitter release from control, hiw ${ }^{N D 8}$, Hiw-rescue, and Hiw $\Delta$ RING-rescue third-instar larvae. Sample traces of spontaneous release and evoked release are shown (Fig. 5a). Presynaptic expression of the wild-type UAS-hiw transgene rescues the deficit in both spontaneous and evoked neurotransmitter release (Fig. 5b,c). The quantal content, which is estimated by dividing the mean EJP amplitude by the mean mEJP amplitude, is also rescued (Fig. $5 d$ ). In contrast to rescue with wild-type UAS-Hiw transgene, the UAS-HiwDRING transgene is unable to rescue either the quantal size or quantal content defects (Fig. 5). These data demonstrate that presynaptic expression of hiw is required for normal physiological function at the Drosophila NMJ and that the RING domain of Hiw plays an essential role in regulating synaptic function.

\section{Neuronal expression of Hiw $\Delta$ RING inhibits hiw function}

Because Hiw functions to restrain synaptic growth, we tested whether excess Hiw in an otherwise wild-type background could lead to a reduction of synapse size. Overexpression of transgenic hiw from either of two neuronal Gal4 drivers [elav-Gal4 or BG380-Gal4 (Yao and White, 1994; Budnik et al., 1996)] did not lead to a decrease in synaptic bouton number (data not shown). In fact, when Hiw is overexpressed from a very strong neuronal driver, ELAV-GeneSwitch (Osterwalder et al., 2001; Roman et al., 2001), there is a tendency toward synaptic overgrowth and a significant decrease of the bouton size (Fig. 6). Because there is no gain-offunction phenotype that is reciprocal to the loss-of-function phenotype, this suggests that endogenous levels of Hiw are sufficient to perform fully the function of Hiw. In fact, the very modest dominantnegative effect from overexpressing wildtype Hiw implies that Hiw may act in a complex whose stoichiometry may be disrupted by excess Hiw protein.

In contrast to the modest effects from overexpression of wild-type Hiw, overexpression of the Hiw $\Delta$ RING transgenic protein results in markedly expanded synapses (Fig. 6a). When driven by the strong ELAV-GeneSwitch driver, expression of Hiw $\Delta$ RING leads to a more than twofold increase in both the number of boutons and the number of branch points, a $50 \%$ increase in synaptic span and a $50 \%$ reduction in bouton size (Fig. $6 b-e$ ). If Hiw $\Delta$ RING is acting as a dominant negative to inhibit endogenous Hiw function, this phenotype should be sensitive to the levels of wild-type Hiw protein. As predicted for a true dominant negative, expression of Hiw $\Delta$ RING in an hiw heterozygous background leads to an additional $56 \%$ increase in the number of boutons $[98.1 \pm 6.0(n=$

a

b
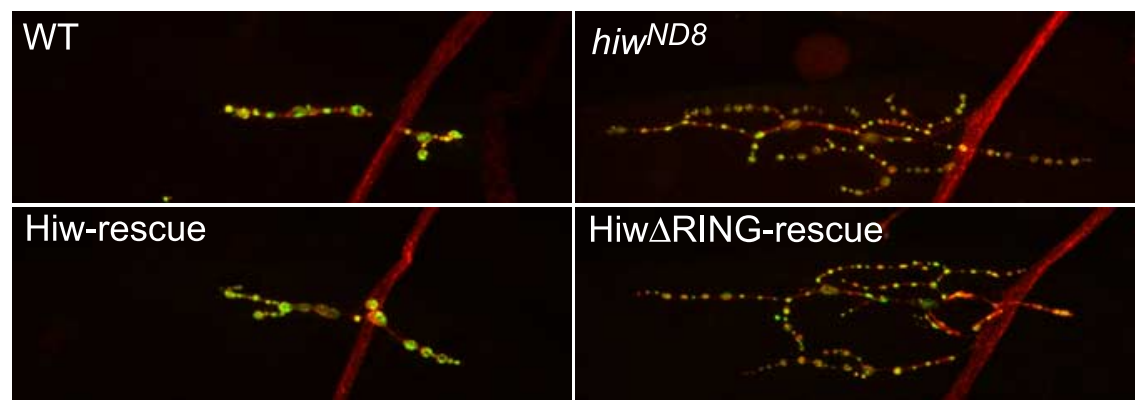

C
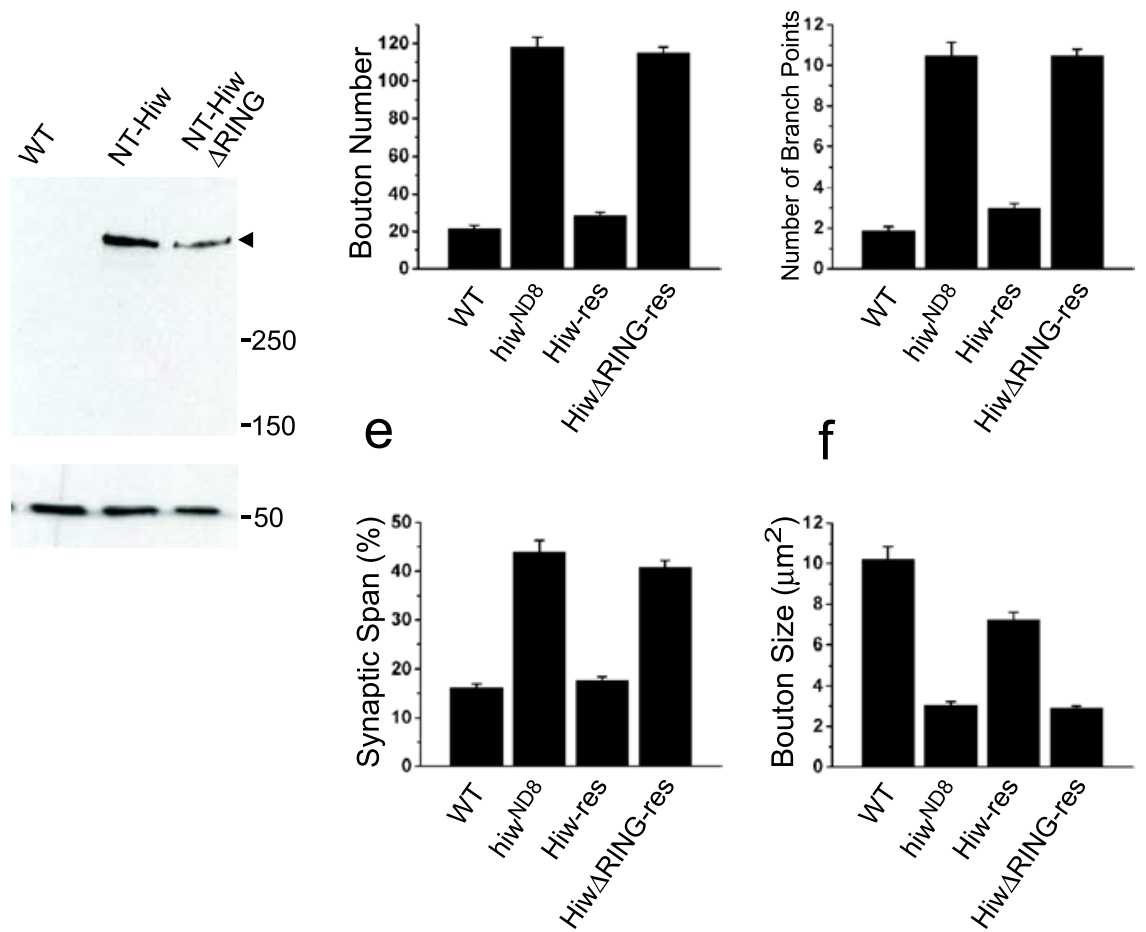

Figure 4. The putative E3 ligase RING finger domain is essential for Hiw function. $\boldsymbol{a}$, Representative confocal images of muscle 4 synapses costained with DVGLUT (green) and Fasll (red), in WT (elav Gal4/+), hiw ${ }^{\text {ND8 }}$, Hiw-rescue (hiw ${ }^{\text {ND8 }} / Y$; UAS-NT-Hiw/+; elav Gal4/+), and Hiw $\Delta$ RING-rescue (hiw ${ }^{N D 8} / Y$; UAS-NT-Hiw $\Delta R I N G /+$; elav Gal4/+) third-instar larvae. $\boldsymbol{b}$, Expression of the transgenic NT-Hiw proteins in the rescue larvae was analyzed by immunoblot with peroxidase-antiperoxidase complex, as shown in the top panel. The arrowhead points to the NT-Hiw band at $\sim 590 \mathrm{kDa}$. The blot in the bottom panel was probed for $\beta$-tubulin as a loading control. The numbers to the right are molecular masses (in kilodaltons). c-f, Quantification of bouton number (c), number of branch points $(\boldsymbol{d})$, synaptic span (e), and bouton size $(\boldsymbol{f})$ in WT, hiw ${ }^{N D 8}$, Hiw-rescue (Hiw-res), and Hiw $\Delta$ RING-rescue third-instar larvae. For bouton number, the number of branch points and synaptic span are $n=31,26,42$, and 50 cells, respectively; for bouton size, $n=15,16,16$, and 19 cells, respectively. Neuronal expression of Hiw $\Delta$ RING failed to rescue the morphological defects of hiw in all parameters $\left(p>0.3\right.$, Hiw $\Delta$ RING-rescue vs hiw $\left.{ }^{N D 8}\right)$. Error bars represent SEM.

$30) ; p \ll 0.001]$ and an additional $42 \%$ increase in the number of branch points [9.1 $\pm 0.7(n=30) ; p<0.005]$, compared with the expression of Hiw $\Delta$ RING in the wild-type background (Fig. 6). We conclude that the Hiw $\Delta$ RING protein acts as a dominant negative and inhibits the function of endogenous protein. Therefore, this mutant protein likely interacts with at least some of the same binding partners as endogenous Hiw. This further supports the conclusion that the RING domain is essential for hiw function. In addition, the selective disruption of Hiw activity presynaptically by a dominant negative demonstrates that not only is 
a

\section{Evoked}

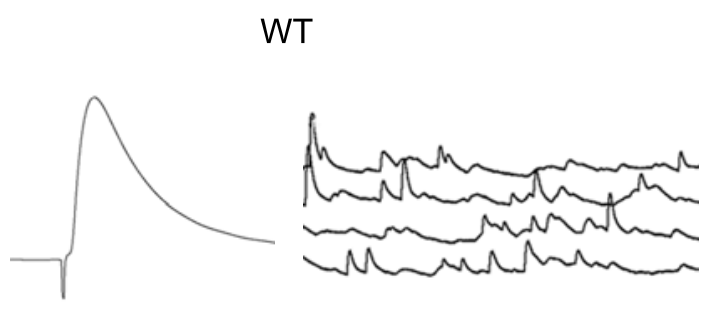

hiw $^{N D 8}$

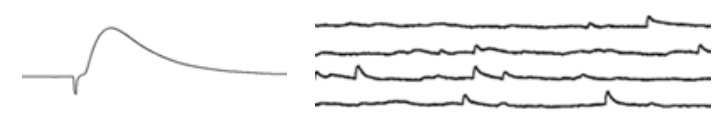

Hiw-res

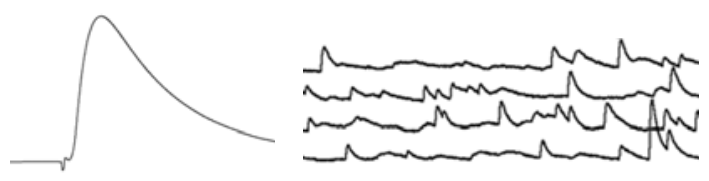

Hiw $\Delta$ RING-res
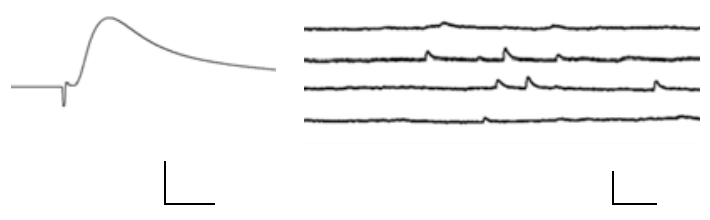

Figure 5. Impaired neurotransmission at hiw mutant NMJs is rescued by presynaptic expression of wild-type Hiw but not Hiw with a disrupted RING finger domain. $\boldsymbol{a}$, Representative traces of evoked and spontaneous transmitter release recorded from WT (elav Gal4/+), hiw ${ }^{\text {ND8 }}$, Hiw-rescue (Hiw-res) (hiw ${ }^{\text {ND8 }} / Y$; UAS-Hiw/+; elav Gal4/+), and Hiw $\Delta$ RING-rescue (hiw ${ }^{N D 8} / Y$; UAS-NTHiw $\Delta R I N G /+$; elav Gal4/+). Calibration: $20 \mathrm{~ms}, 5 \mathrm{mV}$ for evoked release; $200 \mathrm{~ms}, 2 \mathrm{mV}$ for spontaneous release. $\boldsymbol{b}$ - $\boldsymbol{d}$, The mean mEJP amplitude (b), EJP amplitude (c), and quantal content (d) are plotted for WT, hiw ${ }^{N D 8}$, Hiw-rescue, and Hiw $\Delta$ RING-rescue ( $n=19,23,10$, and 11 cells, respectively). The electrophysiological defects in the hiw mutant are rescued in all three parameters by presynaptic expression of Hiw ( $p \ll 0.001$, Hiw-res vs hiw ${ }^{N D 8} ; p>0.1$, Hiw-res vs WT) but not Hiw $\Delta$ RING ( $p>0.5$, Hiw $\Delta$ RING-res vs hiw ${ }^{N D 8}$ ). Error bars represent SEM.

Hiw activity in the presynaptic cell sufficient, as shown by rescue, but it is also necessary.

\section{Temporal requirements for hiw function}

In the hiw mutant, abnormal synaptic overgrowth is evident in the late embryo and persists throughout larval development (Wan et al., 2000). Hence, hiw function may be required throughout development, or, alternatively, hiw function may only be required in the embryo to set cell fate to either a low or high synaptic growth mode. To distinguish between these alternatives, we took advantage of the temporal- and tissue-specific regulation of transgene expression afforded by the GeneSwitch Gal4 system (Osterwalder er at, 2001). GeneSwitch involves a Gal4 fusion protein that should only activate transcription of UAS transgenes after the addition of the progesterone analog
RU486 (Osterwalder er at, 2001). We compared synaptic morphology and function of hiw ${ }^{N D 8}$ mutants expressing ELAVGeneSwitch under four scenarios (Fig. 7): (1) in the absence of the UAS-GFP-Hiw transgene to obtain the baseline hiw phenotype, (2) in the presence of the UASGFP-Hiw transgene and the addition of RU486 during embryogenesis and larval development to assess maximal rescue, (3) in the presence of the UAS-GFP-Hiw transgene but without the addition of RU486 to measure leak in the system, and, finally, (4) in the presence of the UASGFP-Hiw transgene and with the addition of RU486 in the midst of larval development, to assay late roles for Hiw. When GFP-Hiw is expressed throughout development (scenario 2), there is robust rescue of the morphological phenotype with a $77 \%$ reduction in the number of boutons (Fig. 7a). In the absence of RU486 (scenario 3 ), synapses are still extremely overgrown; however, there is apparently some leaky expression because there is a $27 \%$ decrease in the number of synaptic boutons (Fig. 7a). When RU486 is added during larval development (scenario 4), there is an additional $\sim 25 \%$ reduction in bouton number, compared with larvae in the absence of RU486 ( $p<0.005)$. Hence, Hiw can function to regulate synaptic growth during larval development. The inability of Hiw expression in larvae to rescue fully the hiw phenotype demonstrates that Hiw cannot reverse synaptic overgrowth that has already occurred but that it can regulate ongoing synaptic growth. Compared with leaky expression, late expression of Hiw did not lead to a significant decrease in synaptic branching [scenario 1, $6.61 \pm$ $0.32(n=36)$; scenario $2,2.18 \pm 0.19(n=$ $33)$; scenario $3,5.86 \pm 0.47(n=35)$; scenario $4,5.00 \pm 0.35(n=42)(p>0.1$, scenario 3 vs 4$)$ ] or synaptic expansion [scenario $1,43.58 \pm 1.55 \%(n=36)$; scenario $2,17.22 \pm 0.93 \%(n=33)$; scenario $3,32.43 \pm 1.45 \%(n=35)$; scenario 4 , $28.07 \pm 1.16 \%(n=42)(p>0.05$, scenario 3 vs 4$)]$, suggesting that those synaptic phenotypes are primarily determined earlier in development. These data highlight a requirement for Hiw both in the embryo and during larval development and are consistent with an ongoing requirement for hiw function.

We also assessed the temporal requirements for hiwdependent regulation of synaptic function. As with synapse morphology, the presence of RU486 throughout development (scenario 2) leads to a robust rescue of quantal content (Fig. $7 b$ ). Surprisingly, leaky GFP-Hiw expression in the absence of RU486 (scenario 3) led to a nearly complete rescue of the quantal content defect (Fig. 7b). This suggests that regulation of synaptic growth and function may have different dosage requirements for Hiw; very low doses of Hiw are more effective in rescuing synaptic function. Finally, the addition of RU486 during larval develop- 
a
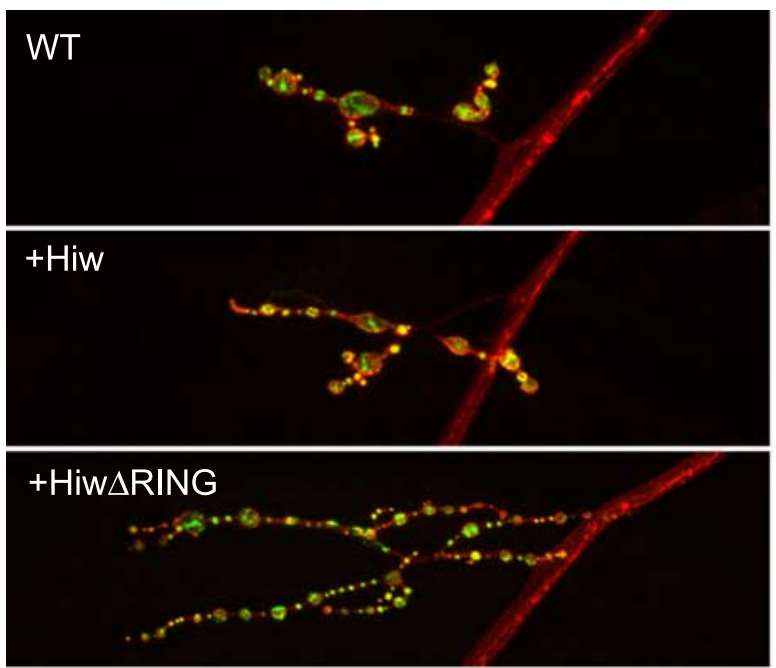

b
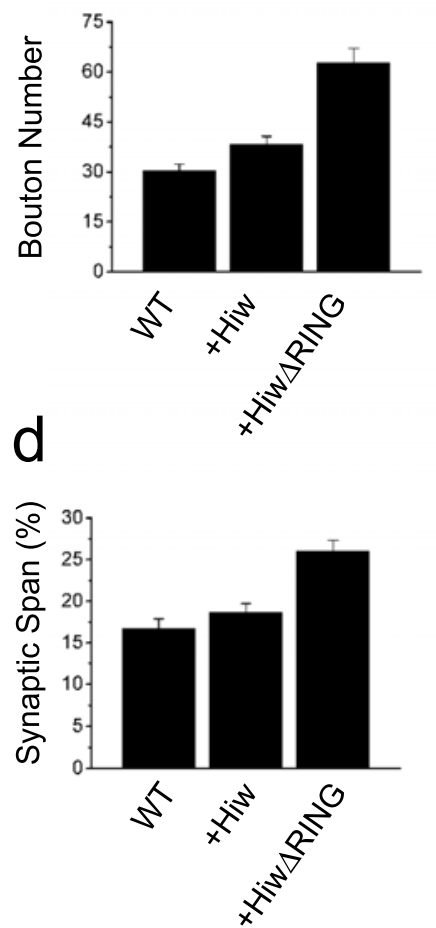

e
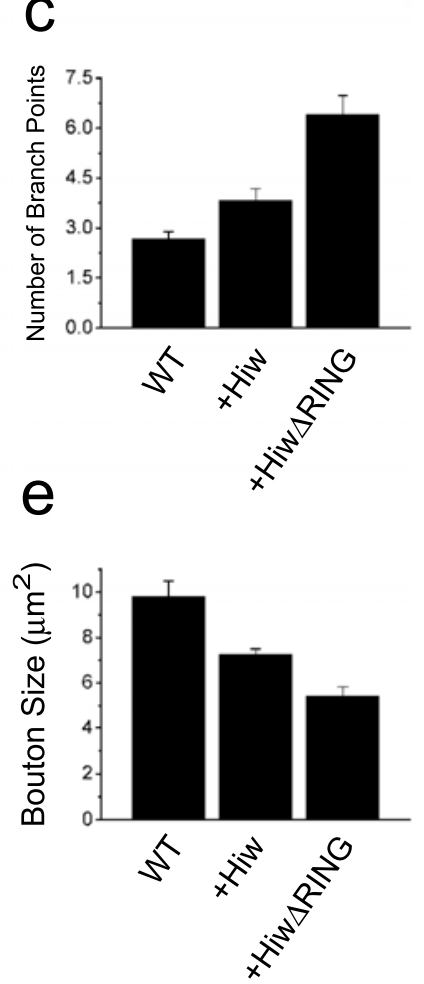

Figure 6. Neuronal expression of Hiw $\Delta$ RING inhibits hiw function. $\boldsymbol{a}$, Representative confocal images of muscle 4 synapses costained with DVGLUT (green) and Fasll (red) in wild-type (ELAVGeneSwitch/+), Hiw overexpression (+Hiw, UAS-NT-Hiw/+; ELAV-GeneSwitch/+), and Hiw $\Delta$ RING overexpressing (+HiwDRING, UAS-NT-Hiw/+; ELAV-GeneSwitch/+) third-instar larvae. $\boldsymbol{b}-\boldsymbol{e}$, Quantification of bouton number $(\boldsymbol{b})$, number of branch points (c), synaptic span $(\boldsymbol{d})$, and bouton size (e) in WT, + Hiw, and + Hiw $\Delta$ RING third-instar larvae. For bouton number, the number of branch points and synaptic span are $n=30,29$, and 29 cells, respectively; for bouton size, $n=18$, 17 , and 17 cells, respectively. Overexpression of Hiw does not significantly change the number of bouton, branch points, and synaptic span ( $p>0.1$, + Hiw vs WT) but leads to a decrease in bouton size ( $p<0.005$, + Hiw vs WT). In contrast, larvae overexpressing Hiw $\Delta$ RING show markedly expanded synapses ( $p \ll 0.001$ for all parameters, + Hiw $\Delta$ RING vs WT). Error bars represent SEM.

ment (scenario 4) leads to a complete rescue of synaptic function, with a significant increase in quantal content when compared with that caused by leaky expression $(p<0.005)$ (Fig. $7 b)$. This result has several implications. First, it provides additional evi-

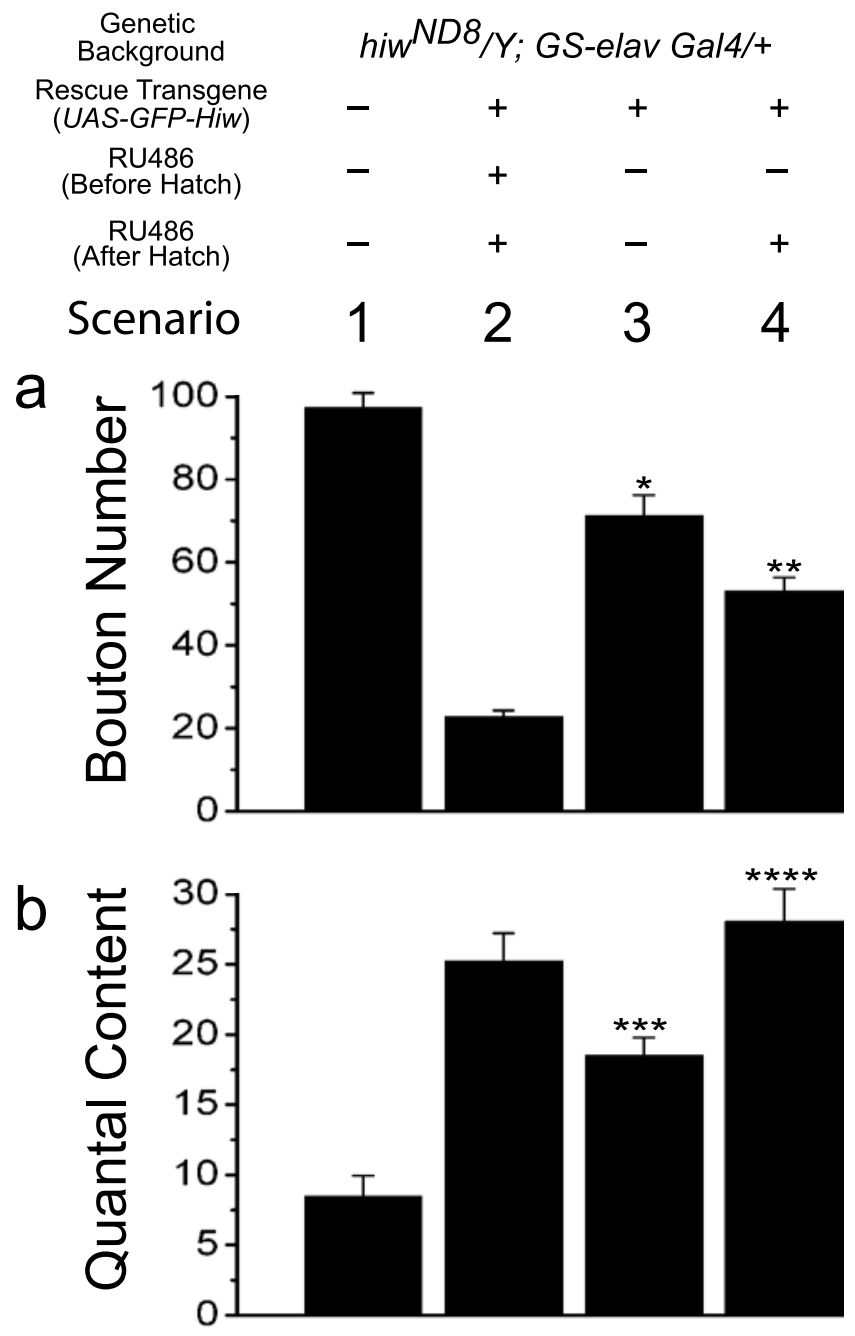

Figure 7. The timing of hiw function in regulating synaptic morphology and physiology. $\boldsymbol{a}$, $\boldsymbol{b}$, The header of the figure describes the genetic background, the presence of rescue transgene, and the manipulation of RU486 in the samples quantified underneath. Synaptic morphology and function were analyzed in four scenarios: (1) baseline $h{ }^{N D 8}$ phenotype, (2) full rescue with RU486, (3) leaked rescue without RU486, and (4) later rescue with RU486 switch. $\boldsymbol{a}$, Quantification of bouton numbers of muscle 4 synapses in the four scenarios $(n=36,33,35$, and 42 cells, respectively). Leaked rescue shows a $27 \%$ reduction in bouton number compared with baseline hiw ${ }^{N D 8}\left({ }^{*} p<0.001\right)$. Later rescue shows an additional $25 \%$ reduction in bouton number compared with leaked rescue $(* * p<0.005)$. $\boldsymbol{b}$, Histogram showing the quantal content for the four scenarios recorded from muscle 6 of third-instar larvae $(n=12,15,13$, and 10 cells, respectively). Leaked rescue shows a twofold increase on the quantal content compared with baseline hiw $^{N D 8}\left({ }^{* * *} p<0.005\right)$. Later rescue shows an additional $52 \%$ increase in quantal content compared with leaked rescue $\left(^{* * *} p<0.005\right)$. Error bars represent SEM.

dence that Hiw continues to function in the larvae. Second, the identification of a genotype with abnormal synapse morphology (a more than twofold increase in bouton number), but normal synaptic function, demonstrates that the morphological and physiological hiw phenotypes are independent. Third, the ability of late Hiw expression to rescue synaptic transmission completely argues that the functional phenotype is not a developmental defect but that Hiw may be a short-term regulator of synaptic transmission.

\section{Discussion}

Highwire is a huge, evolutionarily conserved protein that inhibits synaptic growth and promotes synaptic transmission at the Drosophila neuromuscular junction. To investigate how hiw regu- 
lates synaptic development, we initiated a structure/function analysis. We find that hiw is required exclusively in the presynaptic cell for normal synaptic growth and function. The Hiw RING motif, which is a putative ubiquitin ligase domain, is essential for hiw function. hiw acts throughout larval development to control the structural and functional development of the synapse. Finally, we demonstrate that the morphological and physiological phenotypes of hiw mutants show different sensitivities to the dose and timing of hiw expression, suggesting that hiw may regulate independent pathways controlling synaptic growth and function.

\section{Localization and phenotype of hiw}

Hiw is reported to localize to periactive zones, the regions surrounding but not including the active zone, in presynaptic terminals (Sone et al., 2000; Wan et al., 2000). It has been proposed that the periactive zone is a specialized domain that regulates synaptic growth because at least three proteins important for regulating synaptic growth localize there: Hiw, FasII, and Still life (Sone et al., 1997; Sone et al., 2000). However, the evidence that Hiw localizes to periactive zones comes from staining with the monoclonal antibody $6 \mathrm{H} 4$. We demonstrated that $6 \mathrm{H} 4$ does not recognize Hiw. Synaptic staining with $6 \mathrm{H} 4$ persists in all hiw alleles, including excision mutants that delete the region of the protein against which the antibody was raised. In addition, $6 \mathrm{H} 4$ is unable to recognize misexpressed transgenic Hiw. To determine where Hiw is localized, we generated sera to 10 independent peptide and fusion protein antigens. Although we found a single serum that recognizes Hiw on an immunoblot, we were unable to generate sera that work for immunocytochemistry. As such, we cannot determine the endogenous localization pattern of Hiw. We generated GFP-tagged UAS-GFP-Hiw transgenes that are fully capable of rescuing hiw mutants. The GFP-tagged Hiw does localize to synapses, although it does not extensively colocalize with synaptic vesicles, periactive zones, or active zones (Fig. 3). The transgenic protein does appear to be enriched in synapses, because it is barely detectable in the preterminal axon. However, this overexpressed protein is visible in cell bodies and axon bundles. Similar results are found with GFP-tagged RPM-1, which is detected in synapses, axons, and cell bodies of C. elegans (Schaefer et al., 2000; Zhen et al., 2000). We conclude that Hiw is likely present at synapses.

We previously identified a large number of hiw alleles, including many with stop codons (Wan et al., 2000; DiAntonio et al., 2001). We find here that all of these nonsense alleles express truncated protein and indeed also express some full-length protein attributable to readthrough of the stop codon. This finding raises the possibility that no hiw alleles are true nulls. In fact, both screens that identified hiw alleles selected for viable mutants, which may have biased the screens against true null alleles (Wan et al., 2000; DiAntonio et al., 2001). We have now generated two excision mutants of hiw: one that deletes at least the $\mathrm{N}$-terminal 2784 amino acids $\left(h i w^{\Delta N}\right.$ ), and another that appears to delete the C-terminal 2449 amino acids $\left(\right.$ hiw $\left.^{\Delta C}\right)$. Although either allele could potentially express a truncated but partially functional protein, these are the first alleles that do not express any full-length protein. Like the previously identified alleles, these mutants are viable and have synaptic phenotypes that are essentially identical to a previously characterized genetic null allele, hiw ${ }^{N D 8}$ (Fig. 2). Therefore, the previously described hiw phenotype is likely the null phenotype.

\section{Rescue of hiw}

RNA in situ analysis has demonstrated that hiw is abundantly expressed in the CNS, but that cannot exclude the possibility of lower-level expression in other tissues, such as muscle. In fact, in vertebrates, PAM has been detected in skeletal muscles (Guo et al., 1998). Instead of asking where hiw is expressed, we wished to address where hiw functions. We find that presynaptic hiw is both necessary and sufficient for generating a morphologically and physiologically normal synapse. Postsynaptic expression has no detectable effect on synaptic development. These results are consistent with findings in worms and zebrafish (Schaefer et al., 2000; Zhen et al., 2000; D'Souza et al., 2005). The conserved site of action for hiw supports the view that the mechanism of action of hiw may also be conserved.

It is likely that Hiw and its homologs function as E3 ubiquitin ligases. It contains a RING motif that can function as E3 ubiquitin-protein ligase in vitro (D'Souza et al., 2005), and this domain of the Hiw protein is the most highly conserved across species (Wan et al., 2000). In flies, hiw genetically interacts with the deubiquitinating protease fat facets (DiAntonio et al., 2001), and, in worms, RPM-1 physically interacts with proteins that form an E3 ubiquitin ligase complex (Liao et al., 2004; Nakata et al., 2005). In addition, mutations have been identified in flies and worms that are predicted to disrupt the RING domain; however, these mutant proteins may not be expressed (Schaefer et al., 2000; Wan et al., 2000; Zhen et al., 2000). Therefore, it is unknown whether Hiw ligase activity is necessary for none, some, or all of the functions of hiw at the synapse. We have now expressed an hiw transgene encoding a mutant RING domain. The mutations disrupt two conserved cysteine residues that are required for activity of RING domain ubiquitin ligases (Lorick et al., 1999). This mutant protein is abundantly expressed, but cannot rescue any of the hiw mutant phenotype. Moreover, this RING mutant acts as a potent dominant negative. This suggests that the mutant protein is not grossly misfolded but instead likely interacts with Hiw-binding proteins. This supports the assertion that the RING domain, and ubiquitin ligase activity, is essential for hiw-dependent regulation of both synaptic morphology and transmission.

Because Hiw restrains synaptic growth, we predicted that overexpression of Hiw could reduce synaptic complexity, generating small NMJs with few boutons. We did not observe such a gain-of-function phenotype, indicating that endogenous Hiw levels are not limiting for synaptic growth control. No gain-offunction phenotypes have been reported for $r p m-1$ either (Schaefer et al., 2000; Zhen et al., 2000). These results are consistent with the finding that RPM-1 binds to a multiprotein ubiquitin ligase complex (Liao et al., 2004). Overexpressing a single member of that complex may not lead to increased function. In fact, the very weak dominant-negative phenotype caused by overexpression of wild-type Hiw suggests that excess Hiw may disrupt the stoichiometry of such a functional complex.

\section{Timing of hiw function}

We sought to distinguish between two models for the temporal requirement for hiw function. In hiw mutants, synaptic growth is excessive from the time of initial synapse formation (Wan et al., 2000 ). Hence, hiw may act exclusively during embryogenesis to set cell fate, programming the motoneuron for normal or excessive growth. Alternatively, hiw may function throughout development to regulate the synaptic growth rate of the motoneuron. This second model would allow for modification of synaptic growth during development and would be consistent with a function for hiw in activity-dependent synaptic plasticity. To investi- 
gate the temporal requirements for hiw, we took advantage of the GeneSwitch Gal4 system for tissue-specific and temporally specific control of gene expression (Osterwalder et al., 2001; Roman et al., 2001). We found that expression of hiw after embryogenesis leads to a decrease in synaptic bouton number and a complete rescue of synaptic function. Hence, hiw not only acts in the embryo but can function throughout the larval growth period to control synaptic morphogenesis and transmission. We note that addition of hiw during larval development does not reverse the excess growth that occurred in the embryo and early larval stage. This may explain why similar studies in C. elegans found a requirement for $r p m-1$ early in synaptic development (Schaefer et al., 2000; Zhen et al., 2000). hiw is required early in development, but the quantitative analysis of synaptic growth and function that is feasible at the Drosophila NMJ reveals that hiw also functions later.

As we manipulated the timing of hiw expression, we found differential rescue of the morphological and physiological phenotypes of hiw. When hiw is expressed late, synaptic transmission is fully rescued, although there is still a twofold to threefold increase in bouton number. Hence, the physiological phenotype is not secondary to developmental defects at the synapse. We demonstrated previously that the morphological and physiological phenotypes of hiw are genetically separable, because an hiw, fat facets double mutant shows a partial rescue of physiology with no rescue of morphology (DiAntonio et al., 2001). However, the complete rescue of physiology by the late expression of hiw does more than show that morphology and physiology are independent: it raises the intriguing possibility that hiw functions as a short-term regulator of synaptic function. If hiw has an ongoing role in setting synaptic strength in addition to a developmental role controlling synaptic growth, it is in an ideal position to integrate and coordinate signals that regulate morphological and physiological synaptic plasticity.

\section{References}

Brand AH, Perrimon N (1993) Targeted gene expression as a means of altering cell fates and generating dominant phenotypes. Development 118:401-415.

Budnik V, Koh YH, Guan B, Hartmann B, Hough C, Woods D, Gorczyca M (1996) Regulation of synapse structure and function by the Drosophila tumor suppressor gene dlg. Neuron 17:627-640.

Burgess RW, Peterson KA, Johnson MJ, Roix JJ, Welsh IC, O’Brien TP (2004) Evidence for a conserved function in synapse formation reveals Phrl as a candidate gene for respiratory failure in newborn mice. Mol Cell Biol 24:1096-1105.

Daniels RW, Collins CA, Gelfand MV, Dant J, Brooks ES, Krantz DE, DiAntonio A (2004) Increased expression of the Drosophila vesicular glutamate transporter leads to excess glutamate release and a compensatory decrease in quantal content. J Neurosci 24:10466-10474.

DiAntonio A, Haghighi AP, Portman SL, Lee JD, Amaranto AM, Goodman CS (2001) Ubiquitination-dependent mechanisms regulate synaptic growth and function. Nature 412:449-452.

D’Souza J, Hendricks M, Le Guyader S, Subburaju S, Grunewald B, Scholich K, Jesuthasan S (2005) Formation of the retinotectal projection requires Esrom, an ortholog of PAM (protein associated with Myc). Development 132:247-256.

Guo Q, Xie J, Dang CV, Liu ET, Bishop JM (1998) Identifcation of a large Myc-binding protein that contains RCC1-like repeats. Proc Natl Acad Sci USA 95:9172-9177.

Hoang B, Chiba A (2001) Single-cell analysis of Drosophila larval neuromuscular synapses. Dev Biol 229:55-70.

Joazeiro CA, Weissman AM (2000) RING finger proteins: mediators of ubiquitin ligase activity. Cell 102:549-552.
Liao EH, Hung W, Abrams B, Zhen M (2004) An SCF-like ubiquitin ligase complex that controls presynaptic differentiation. Nature 430:345-350.

Lorick KL, Jensen JP, Fang S, Ong AM, Hatakeyama S, Weissman AM (1999) RING fingers mediate ubiquitin-conjugating enzyme (E2)-dependent ubiquitination. Proc Natl Acad Sci USA 96:11364-11369.

Marrus SB, DiAntonio A (2004) Preferential localization of glutamate receptors opposite sites of high presynaptic release. Curr Biol 14:924-931.

Marrus SB, Portman SL, Allen MJ, Moffat KG, DiAntonio A (2004) Differential localization of glutamate receptor subunits at the Drosophila neuromuscular junction. J Neurosci 24:1406-1415.

McCabe BD, Hom S, Aberle H, Fetter RD, Marques G, Haerry TE, Wan H, O'Connor MB, Goodman CS, Haghighi AP (2004) Highwire regulates presynaptic BMP signaling essential for synaptic growth. Neuron 41:891-905.

Murthy V, Han S, Beauchamp RL, Smith N, Haddad LA, Ito N, Ramesh V (2004) Pam and its ortholog highwire interact with and may negatively regulate the TSC1.TSC2 complex. J Biol Chem 279:1351-1358.

Nakata K, Abrams B, Grill B, Goncharov A, Huang X, Chisholm AD, Jin Y (2005) Regulation of a DLK-1 and p38 MAP kinase pathway by the ubiquitin ligase RPM-1 is required for presynaptic development. Cell 120:407-420.

Osterwalder T, Yoon KS, White BH, Keshishian H (2001) A conditional tissue-specific transgene expression system using inducible GAL4. Proc Natl Acad Sci USA 98:12596-12601.

Parker L, Gross S, Alphey L (2001) Vectors for the expression of tagged proteins in Drosophila. Biotechniques 31:1280-1282, 1284, 1286.

Petersen SA, Fetter RD, Noordermeer JN, Goodman CS, DiAntonio A (1997) Genetic analysis of glutamate receptors in Drosophila reveals a retrograde signal regulating presynaptic transmitter release. Neuron 19:1237-1248.

Pierre SC, Häusler J, Birod K, Geisslinger G, Scholich K (2004) PAM mediates sustained inhibition of cAMP signaling by sphingosine-1-phosphate. EMBO J 23:3031-3040.

Rigaut G, Shevchenko A, Rutz B, Wilm M, Mann M, Seraphin B (1999) A generic protein purification method for protein complex characterization and proteome exploration. Nat Biotechnol 17:1030-1032.

Roman G, Endo K, Zong L, Davis RL (2001) P[Switch], a system for spatial and temporal control of gene expression in Drosophila melanogaster. Proc Natl Acad Sci USA 98:12602-12607.

Schaefer AM, Hadwiger GD, Nonet ML (2000) rpm-1, a conserved neuronal gene that regulates targeting and synaptogenesis in C. elegans. Neuron 26:345-356.

Scholich K, Pierre S, Patel TB (2001) Protein associated with Myc (PAM) is a potent inhibitor of adenylyl cyclases. J Biol Chem 276:47583-47589.

Seedorf M, Damelin M, Kahana J, Taura T, Silver PA (1999) Interactions between a nuclear transporter and a subset of nuclear pore complex proteins depend on Ran GTPase. Mol Cell Biol 19:1547-1557.

Sone M, Hoshino M, Suzuki E, Kuroda S, Kaibuchi K, Nakagoshi H, Saigo K, Nabeshima Y, Hama C (1997) Still life, a protein in synaptic terminals of Drosophila homologous to GDP-GTP exchangers. Science 275:543-547.

Sone M, Suzuki E, Hoshino M, Hou D, Kuromi H, Fukata M, Kuroda S, Kaibuchi K, Nabeshima Y, Hama C (2000) Synaptic development is controlled in the periactive zones of Drosophila synapses. Development 127:4157-4168.

Stewart BA, Atwood HL, Renger JJ, Wang J, Wu CF (1994) Improved stability of Drosophila larval neuromuscular preparations in haemolymphlike physiological solutions. J Comp Physiol A Neuroethol Sens Neural Behav Physiol 175:179-191.

Wan HI, DiAntonio A, Fetter RD, Bergstrom K, Strauss R, Goodman CS (2000) Highwire regulates synaptic growth in Drosophila. Neuron 26:313-329.

Yao KM, White K (1994) Neural specificity of elav expression: defining a Drosophila promoter for directing expression to the nervous system. J Neurochem 63:41-51.

Zhen M, Huang X, Bamber B, Jin Y (2000) Regulation of presynaptic terminal organization by C. elegans RPM-1, a putative guanine nucleotide exchanger with a RING-H2 finger domain. Neuron 26:331-343.

Zinsmaier KE, Hofbauer A, Heimbeck G, Pflugfelder GO, Buchner S, Buchner E (1990) A cysteine-string protein is expressed in retina and brain of Drosophila. J Neurogenet 7:15-29. 